\title{
EpidemiXs: Harnessing digital technology in the fight against COVID-19 and the associated infodemic
}

\author{
Jeannine Lemaire ${ }^{\mathrm{a}, *}$, Elsa Ramil ${ }^{\mathrm{a}}$, Veronique Ines Thouvenot ${ }^{\mathrm{b}, *}$ and Jordi Serrano Pons ${ }^{\mathrm{a}}$ \\ ${ }^{\text {a }}$ UniversalDoctor, Milan, Italy \\ ${ }^{\mathrm{b}}$ Millennia2025 Women and Innovation Foundation, Lyon, France
}

Received 20 October 2021

Accepted 2 November 2021

\begin{abstract}
.
BACKGROUND: EpidemiXs is an innovative ecosystem of digital tools centralizing official and validated information on COVID-19 for healthcare workers and the general public in a single hub.

OBJECTIVE: The vision of EpidemiXs is to foster collaboration between researchers, institutions and individuals to promote "open data" in order to enrich the scientific community and further accelerate science in the fight against COVID-19.

METHODS: Through its set of solutions, EpidemiXs Info, EpidemiXs TV and EpidemiXs Studies, this innovative ecosystem contributes to advancing collaborations, data collection and analysis, and helps find funders.

RESULTS: EpidemiXs was launched in March 2020 in Spain with 30 healthcare institutions and rapidly reached close to 1 million users and 2 million views. EpidemiXs gained international recognition when it was awarded the Barcelona Health Hub Awards (BHHAwards) 2020 of the category "Best Startup Initiative to help tackle COVID-19".

CONCLUSION: EpidemiXs has proven the efficiency of the rapid deployment of digital tools in times of COVID-19.
\end{abstract}

Keywords: COVID-19, infodemic, pandemic, digital health, technology, research, open data, healthcare

\section{Introduction}

As the coronavirus disease (COVID-19) pandemic unfolded, a growing infodemic was also taking place, a phenomenon coined by the World Health Organization (WHO) as an overabundance of information, including the spread of both misinformation and disinformation, which made it difficult for people to find trustworthy sources and reliable guidance. Social media and communication platforms became amplifiers of the infodemic, putting key measures to control the spread of COVID-19, such as mask-wearing recommendations and immunization campaigns, at risk. At the same time, there was a lack of data to inform evidence-based policies and strategies to control the pandemic. These phenomena combined led to dangerous cycles of poor observance of public health measures and jeopardized the global response to the pandemic.

\footnotetext{
${ }^{*}$ Corresponding authors: Jeannine Lemaire, UniversalDoctor, Milan, Italy. E-mail: jlemaire@ universaldoctor.com. Veronique Ines Thouvenot, Millennia2025 Women and Innovation Foundation, Lyon, France. E-mail: Thouvenot.veronique@ millennia2025foundation.org.
} 


\subsection{EpidemiXs, an ecosystem of digital tools}

The impact was not only felt by the general public, but also the medical and healthcare professional community. In Spain and other localizations around the globe, the team at UniversalDoctor, a social enterprise founded by a medical doctor to co-create digital global health solutions with key stakeholders, began brainstorming with health care workers and citizens in March of 2020, to co-design a set of digital solutions to support communities and local actors in the fight against COVID-19. This led to the birth of the EpidemiXs ecosystem of digital tools to harness technology in the fight against COVID-19 and the associated infodemic.

The EpidemiXs solutions harnessed and leveraged over a decade of the development of different technologies and cross-sector expertise of UniversalDoctor working with the leading public health actors on the global level, such as the WHO, and local level, including regional and local healthcare institutions.

EpidemiXs was first launched in Spain in partnership with 30 healthcare institutions, reaching close to 1 million users and over 2 million views in the first week, and has been deployed in France in collaboration with the Millennia2025 Women and Innovation Foundation for vulnerable populations and healthcare professionals serving them.

\subsection{EpidemiXs Info web app}

The first solution developed was EpidemiXs Info, an accessible and real-time updated web app cocreated by UniversalDoctor with 30 local and international healthcare institutions, to centralize the latest official and validated information, protocols and guidance on COVID-19 from different sources and deliver it through customized portals for HCWs and the general public. Each portal offered information that was suitable for the target audience, allowing them to access dedicated verified information in a quick and easy way.

EpidemiXs Info was supported by a 24/7 dedicated Task Force of over 100 medical experts that investigated, curated, vetted and organized the information on a daily basis in Spanish and French. The Web App rapidly gained international recognition at the Barcelona Health Hub Awards 2020 as the "Best Startup Initiative to help tackle COVID-19".

\subsection{EpidemiXs $T V$}

In parallel, EpidemiXs TV was launched, offering live interviews with key experts from different sectors, civil society and local actors around the world facilitating cross-sector knowledge-sharing across borders and providing the live audiences with access to these experts for their queries. With 29 episodes and a total of 40 hours in Spanish, English and French, EpidemiXs TV offers a wide range of views and expertise on COVID-19 from around the world, including France, United Kingdom, Ethiopia, Mozambique, Russia, USA, Brazil, Argentina, Uruguay, Chile, Central Americas and punctual interventions from many more countries. More than 100 experts participated respecting gender equity in most episodes and reaching about 8.000 views. EpidemiXs TV covered the themes of innovation in times of COVID-19, chronic diseases, mental health, reaching the most vulnerable populations, mobile apps in health and the right to connectivity. All TV episodes are available on Youtube and are planned to serve as educational materials in the future.

\subsection{EpidemiXs Studies}

In response to the lack of Real-World (RWD) Data on COVID-19, EpidemiXs Studies was created 
to empower researchers and healthcare institutions with a data collection and management platform to quickly and easily deploy studies to help build the evidence-based on COVID-19.

The objective was to use the traction of EpidemiXs Info and connect its users to the open studies by researchers in EpidemiXs Studies to rapidly grow the open evidence-base that can be used by institutions and researchers to inform their work on COVID-19.

The first set of studies was launched under the umbrella of Studies4COVID19, in partnership with the primary care center EAP Sardenya in Barcelona, for the first serological testing study in Spain on HCWs and the general public. The data collection system started out with form templates collecting serological prevalence data, symptoms and PCR results, and expanded to collect data points from varying topics from long-COVID to healthcare workers (HCWs) mental health. Through the EpidemiXs Studies data collection platform, thousands of data points have been collected across the eight studies being conducted at four different healthcare institutions across Spain and Andorra. These studies ranged from serological studies to immunological follow-up studies on healthcare workers and the general population, to social studies to better understand and support the grieving process of people who had lost loved ones due to COVID-19.

At its core, EpidemiXs Studies is a platform that connects researchers, potential study participants, institutions, funders, and scientists. In addition to the data collection and management tools, it also offers a dissemination platform to expand the scope of the studies, thereby promoting collaboration between research centers and institutions with the general population. Since its launch, the EpidemiXs Studies dissemination platform has published and shared the work of over 150 COVID-19 related studies in easy-to-understand and user-friendly formats, making the studies and their work more accessible by the general public and driving engagement. The studies included clinical trials, epidemiological studies and lab research studies, which were published with the goal of reaching the general population. Moreover, some studies were actively seeking funding, and EpidemiXs Studies facilitated access to these fundraising campaigns, allowing the general public to further engage with and support the studies. Other studies were actively searching for participants to be a part of their study, therefore EpidemiXs Studies facilitated participant recruitment as well. Almost half of the studies published were co-led by a mix of male and female investigators, and almost $40 \%$ were international studies. A second wave of these tools is being developed now and prepared to be launched under the umbrella of UhDA: Universal Health Digital Access, a new spin-off of UniversalDoctor.

\section{Conclusion}

EpidemiXs has proven the efficiency of the rapid deployment of digital tools in times of COVID-19, starting with the publication of verified information for citizens and healthcare professionals, TV episodes with experts from around the world and the extension to research studies. Mutual collaboration with trusted partners has fostered innovation at a unique pace. Still a lot needs to be learned from the COVID-19 crisis, but yet, the fantastic dedication of the entire teams across different sectors, geographical locations and disciplines will remain a unique experience for all.

\section{Acknowledgments}

This work is a joint reflection with the founders and teams of UniversalDoctor and Millennia2025 Women and Innovation Foundation after the presentation at the third international conference on quality in medicine, global health and law, Paris and online, 14-16 June 2021. 
The authors are grateful for the invitation to participate at the conference to Prof. Christine Huttin, University Aix Marseille, member of the High Council of Public Health, France and Dr. Magdalena Sajdak, Polish Academy of Sciences Scientific Center in Paris, Poland.

\section{Conflict of interest}

None to report. 